\title{
Receptor-mediated endocytosis and trafficking between endosomal- lysosomal vacuoles in Giardia lamblia
}

\author{
Maria R Rivero ${ }^{\mathrm{a}, \dagger}$, Ignacio Jausoro ${ }^{\mathrm{a}}$, Mariano Bisbala ${ }^{\mathrm{a}}$, Constanza Feliziani ${ }^{\mathrm{a}}$, Adriana \\ Lanfredi-Rangel ${ }^{b}$, and Maria C Touz ${ }^{a}{ }^{*}$ \\ alnstituto de Investigación Médica Mercedes y Martín Ferreyra, INIMEC - CONICET, Universidad \\ Nacional de Córdoba, Friuli 2434, Córdoba, Argentina \\ bServiço de Microscopia Eletrônica, Centro de Pesquisas Gonçalo Moniz, FIOCRUZ-BA, Brazil
}

\section{Abstract}

The early branching Giardia lamblia has highly polarized vacuoles, located underneath the plasma membrane, which have at least some of the characteristics of endosomes and of lysosomes. These peripheral vacuoles (PVs) are necessary for nutrient uptake and the maintenance of plasma membrane composition, but whether they carry out sorting and segregation of receptors and ligands is a matter of debate. Here, we showed that the internalization of low-density lipoprotein (LDL) to the PVs is highly dynamic in trophozoites with a rate similar to the internalization of the Low-Density Lipoprotein Receptor-Related Protein 1 (LRP1). Moreover, by analyzing receptormediated and fluid phase endocytosis in living cells, we showed that after endocytosis LDL but not dextran moved laterally between the PVs. We speculate on PV functional heterogeneity and maturation in this parasite.

\section{Keywords}

endocytosis; BODIPY-LDL; TIRFM; uptake visualization; living trophozoites; Giardia lamblia

\section{INTRODUCTION}

The dynamic nature of the endocytic apparatus of mammalian cells has become increasingly clear over the past years. Structures collectively referred to as endosomes are at the crossroads of traffic with the plasma membrane and with the degradative pathway leading to lysosomes. The protozoa parasite Giardia lamblia lacks compartments that resemble early/ late endosomes and lysosomes; instead, trophozoites possess peripheral vacuoles (PVs) that seem to function as endosomes and lysosomes at the same time (Lanfredi-Rangel et al. 1998; Rivero et al. 2011; Rivero et al. 2010; Touz et al. 2003). These vacuoles are acidic, as demonstrated by the uptake of acridine orange and the lysosomal markers LysoSensor and LysoTracker (Lanfredi-Rangel et al. 1998; Touz et al. 2003; Touz et al. 2002), with the presence of hydrolase activities in the PVs, such as acid phosphatase, cysteine proteases, and

\footnotetext{
*Corresponding author: Maria Carolina Touz, Instituto de Investigación Médica Mercedes y Martín Ferreyra, INIMEC - CONICET, Friuli 2434, 5000, Cordoba, Argentina. Phone/fax: (54) (351) 468-1465/54-351-4695163. ctouz@immf.uncor.edu.

$\dagger^{\dagger}$ Current address: Dto. de Cs. Naturales, Fac. de Cs. Exactas Fisicoquímicas y Naturales, Univ. Nac. de Rio Cuarto, Argentina. AUTHORS' CONTRIBUTIONS

MRR carried out the uptake experiments and drafted the manuscript. IJ and MB participated in the design of the study and performed the microscopy analysis. CF and ALR performed the electron microscopy. MCT conceived the study and helped to draft the manuscript. All authors read and approved the final manuscript.
}

CONFLICTS OF INTEREST: None. 
RNases, indicating their lysosomal characteristics (Feely and Dyer 1987; Lindmark 1988; Touz et al. 2002; Ward et al. 1997). They have a potential role in endocytosis, as demonstrated by the uptake of exogenous ferritin and Lucifer yellow, as well as LDL and chylomicrons (Lanfredi-Rangel et al. 1998; Rivero et al. 2011; Rivero et al. 2010). Pulsechase experiments with horseradish peroxidase and fluorescent dextran showed an early and persistent labeling of the PVs, suggesting that there is not distinction between early and late endocytic vesicles in Giardia in contrast to what occurs in higher eukaryotes (Gaechter et al. 2008; Lanfredi-Rangel et al. 1998). Also, we showed that a type I membrane homologous to LRP1 and LRP1b was responsible for the uptake of cholesterol via its interaction with LDL and chylomicron (Rivero et al. 2011). Although we showed that receptor-mediated endocytosis takes place in this parasite (Rivero et al. 2011; Rivero et al. 2010), no time-lapse microscopy in live organisms has been performed to test this event. In this work, we combined total internal reflection fluorescence microscopy (TIRFM) with epifluorescence microscopy to directly study the capture and internalization of fluorescent endocytic markers in living trophozoites. This approach allowed us to observe LDL at the plasma membrane of Giardia, later internalized to vacuoles located just below the plasma membrane. The objective of the present study was to examine whether the endocytosis of LDL through its receptor show homogeneous and steady PV distribution, as was observed for fluid-phase endocytosis of dextran.

\section{MATERIALS AND METHODS}

\section{Giardia Cell Line}

Trophozoites of the isolate WB, clone 1267 (Nash et al. 1988), were cultured in TYI-S-33 medium supplemented with $10 \%$ adult bovine serum and $0.5 \mathrm{mg} / \mathrm{ml}$ bovine bile, as previously described (Keister 1983).

\section{Reagents}

BODIPY-LDL was purchased from Molecular Probes-Invitrogen. 20.000 MW-FITCDextran was purchased from Sigma-Aldrich. Polylysine was purchased from Sigma-Aldrich.

\section{Transmission electron microscopy}

The trophozoites were fixed when still adhered to culture tubes and treated as described (Lanfredi-Rangel et al. 1998).

\section{Epifluorescence and Total Internal Reflection Fluorescence Microscopy (TIRFM)}

Trophozoites were grown in complete medium, suspended in $1 \mathrm{ml}$ of labeling buffer (50 $\mathrm{mM}$ glucose, $10 \mathrm{mM}$ cysteine, $2 \mathrm{mM}$ ascorbic acid in PBS, pH 7.2), and attached to polylysine-treated cover slips for $30 \mathrm{~min}$ at $37^{\circ} \mathrm{C}$. BODIPY-LDL $(7.5 \mu \mathrm{g})$ was loaded before chamber assembly. For epifluorescence and TIRF microscopy, live trophozoites were observed using a 60X 1.45 numerical aperture objective equipped for through-the-objective TIRF illumination using a $488 \mathrm{~nm}$ argon laser on a Nikon TE2000-U microscope with filter cubes optimized for fluorescein/GFP (Chroma Technology, Rockingham, VT). Images were captured with a cooled CCD ORCA II-ER (Hamamatsu) camera and MetaMorph software (Molecular Devices). The uptake events were captured $5 \mathrm{~min}$ post-BODYPI-LDL addition, with images being taken at the rate of 1 frame per 5 seconds. The same imaging parameters were used to photograph trophozoites without BODIPY-LDL as a control for autofluorescence (not shown). Other controls include the use of FITC-dextran (does not adsorb to the surface) and BODIPY-LDL addition to fixed trophozoites (does absorb to the surface but is not internalized). These experiments were performed in triplicate. Live trophozoites were observed using a 60X 1.45 numerical aperture objective equipped for 
through-the-objective TIRF illumination using a $488 \mathrm{~nm}$ argon laser on a Nikon TE2000-U microscope with filter cubes optimized for fluorescein/GFP (Chroma Technology, Rockingham, VT). Images were captured with a cooled CCD ORCA II-ER (Hamamatsu) camera and MetaMorph software (Molecular Devices).

\section{RESULTS AND DISCUSSION}

\section{The PVs are highly polarized vesicles involved in nutrient endocytosis}

By transmission electron microscopy, PVs were identified by the presence of electrontranslucent cores, showing approximately $100-400 \mathrm{~nm}$ in width (Fig. 1A and B) (Feely and Dyer 1987; Kattenbach et al. 1991; Lanfredi-Rangel et al. 1998). When the trophozoite attaches to the host epithelium, the ventral surface of the trophozoite comes in contact with the microvillus border but since organisms are trapped between villi, both ventral and dorsal surface may be adjacent to the host cell. In the trophozoite, we observed the distribution of the PVs along both dorsal and ventral surfaces (Fig. 1B), which is consistent with their role in nutrient uptake and digestion (Lanfredi-Rangel et al. 1998; Rivero et al. 2011; Rivero et al. 2010). Their definitive role as endosomes was recently demonstrated by the PV localization of LDL after internalization via receptor-mediated endocytosis (Fig. 1C) (Rivero et al. 2011; Rivero et al. 2010). Since PVs were first described, there was speculation on whether the variability in shape and contents might imply difference in their nature, as they act at different steps during the process of protein sorting and degradation. The current study suggests that specific molecules are selectively directed to the PVs through an unusual endocytic mechanism.

\section{LDL is concentrated and internalized in a dynamic process}

TIRFM is a powerful technique to examine whether a receptor is involved in endocytosis, by looking at the kinetics of the internalization of the fluorescent molecules in living organisms. TIRFM principles are based on highly specific fluorescent excitation in a thin specimen region adjacent to the interface between a specimen and a glass coverslip. The total reflection of the illuminating beam generates images of a $\sim 100 \mathrm{~nm}$ region from the glass surface. Visualization and study of ligand-receptor interactions, vesicle release, cell adhesion, and secretion process are some applications of this microscopy technique (Axelrod 2003) (Fig. S1). Recently, TIRFM was used to study the structures involved in the attachment and detachment of Giardia trophozoites (House et al. 2011). However, once attached to cover glasses, the trophozoites detached spontaneously, making it difficult to analyze either molecular and organelle movement. In this work, we performed an adaptation of TIRFM used for neurons (Bisbal et al. 2008; Dupraz et al. 2009) to living Giardia trophozoites, in which cysteine and ascorbic acid were added to the labeling buffer, improving the attachment of the cells at $37^{\circ} \mathrm{C}$ to cover glasses previously treated with polylysine. By using LDL labeled with BODIPY (boron-dipyrromethene), the dynamics of LDL capture and endocytosis were traced. In other cells, it was shown that BODIPY-LDL fluorescence is neither quenched nor reduced by acidic $\mathrm{pH}$ as in the lysosomes to which LDL-bound to receptor traffics, and it can be used to track LDL surface binding and internalization (Goldstein et al. 1983). Therefore, in order to examine the kinetics of the LDL receptor (Giardia lamblia receptor-related protein, GILRP) we followed the internalization of LDL in living Giardia trophozoites. The dynamics of LDL capture and endocytosis were then traced by TIRFM. Internalization events can be detected semiautomatically using maximum pixel intensity and centroid analysis to define sites of insertion (Axelrod 2003). After the addition of BODIPY-LDL, the 488-nm laser was used to visualize the fluorescence in living trophozoites, and the image was processed by displaying a pseudocolor (M1). Within seconds of incubation, the plasma membrane became homogeneously labeled with BODIPY-LDL and bright spots appeared soon after, 
suggesting that LDL was bound to GILRP forming clusters (Fig. 2a). The LDL signal then decreased (Fig. $2 \mathrm{~b}$ to $2 \mathrm{f}$ ), with a half-life of $30 \pm 0.33 \mathrm{~s}$. As expected, the control, adding BODIPY-LDL to fixed trophozoites, showed that LDL was adsorbed to the surface but not internalized (M2). FITC-dextran did not show surface localization or cluster formation by TIRFM (not shown), but internalization by epifluorescence has been reported (Rivero et al. 2010) (see also Fig. 3B). Our results showed that the rate of LDL endocytosis was comparable to the kinetics of LRP1 internalization, a member of the LDL receptor family widely expressed in several tissues (Bu et al. 1994).

\section{LDL and dextran are differentially distributed in the PVs}

Analysis using time-lapse epifluorescence showed that LDL was delivered to the PVs just after the disappearance of the LDL signal in the evanescent wave (M3). Artificial coloring of each frame made it possible to see that, after internalization, BODIPY-LDL moved laterally over time (Fig. 3A) (Touz 2012). Conversely, when the same assay was performed using FITC-dextran, no movement of dextran between vacuoles was observed (Fig. 3B). It is possible that, after formation of coated vesicles and release from the plasma membrane, BODIPY-LDL might be delivered to certain PVs, later being redirected to the definitive vesicle, where it will be separated from the receptor and processed. This lateral movement of BODIPY-LDL raises several questions regarding not only how an LDL molecule behaves but whether there is a maturation process of PVs in Giardia. However, whether this may reflect a distinct function for a group of PVs still remains to be elucidated.

\section{CONCLUSION}

Eukaryotic cells differentiated during evolution, adapting to their increasingly complex environment by acquiring new abilities for nutrient uptake, internalization of growth factors and entry of pathogens, among others. Although the complex array of endosomal/lysosomal compartments responsible for these activities were preserved as modules that perform specific functions in modern eukaryotic cells, the variety and simplicity of these systems in early branching eukaryotes is only starting to become apparent. Thus Giardia possesses organelles that are similar to endosomes and lysosomes (the PVs), which may represent an ancient organellar system that later subdivided into compartments: early and late endosomes, and lysosomes. From in vivo studies of endocytosis events using adapted microscopy techniques, more precise information was acquired leading to a better understanding of the exact role of endocytosis in Giardia pathogenesis and survival.

\section{Supplementary Material}

Refer to Web version on PubMed Central for supplementary material.

\section{Acknowledgments}

The project was supported by Grant Number R01TW00724 from the Fogarty International Center. The content is solely the responsibility of the authors and does not necessarily represent the official views of the Fogarty International Center or the National Institutes of Health. This research was also supported in part by the Argentine Agencia Nacional para la Promoción de la Ciencia y Tecnología (FONCyT- PICT698).

\section{References}

Axelrod D. Total internal reflection fluorescence microscopy in cell biology. Methods Enzymol. 2003; 361:1-33. [PubMed: 12624904]

Bisbal M, Conde C, Donoso M, Bollati F, Sesma J, Quiroga S, Diaz Anel A, Malhotra V, Marzolo MP, Caceres A. Protein kinase d regulates trafficking of dendritic membrane proteins in developing neurons. J Neurosci. 2008; 28:9297-9308. [PubMed: 18784310] 
Bu G, Maksymovitch EA, Geuze H, Schwartz AL. Subcellular localization and endocytic function of low density lipoprotein receptor-related protein in human glioblastoma cells. J Biol Chem. 1994; 269:29874-29882. [PubMed: 7961982]

Dupraz S, Grassi D, Bernis Me, Sosa L, Bisbal M, Gastaldi L, Jausoro I, Caceres A, Pfenninger KH, Quiroga S. The TC10-Exo70 complex is essential for membrane expansion and axonal specification in developing neurons. J Neurosci. 2009; 29:13292-13301. [PubMed: 19846717]

Feely DE, Dyer JK. Localization of acid phosphatase activity in Giardia lamblia and Giardia muris trophozoites. J Protozool. 1987; 34:80-83. [PubMed: 3572844]

Gaechter V, Schraner E, Wild P, Hehl AB. The single dynamin family protein in the primitive protozoan Giardia lamblia is essential for stage conversion and endocytic transport. Traffic. 2008; 9:57-71. [PubMed: 17892527]

Goldstein JL, Basu SK, Brown MS. Receptor-mediated endocytosis of low-density lipoprotein in cultured cells. Methods Enzymol. 1983; 98:241-260. [PubMed: 6321901]

House SA, Richter DJ, Pham JK, Dawson SC. Giardia flagellar motility is not directly required to maintain attachment to surfaces. PLoS Pathog. 2011; 7:e1002167. [PubMed: 21829364]

Kattenbach WM, Pimenta PF, De Souza W, Pinto Da Silva P. Giardia duodenalis: a freeze-fracture, fracture-flip and cytochemistry study. Parasitol Res. 1991; 77:651-658. [PubMed: 1805207]

Keister DB. Axenic culture of Giardia lamblia in TYI-S-33 medium supplemented with bile. Trans R Soc Trop Med Hyg. 1983; 77:487-488. [PubMed: 6636276]

Lanfredi-Rangel A, Attias M, De Carvalho TM, Kattenbach WM, De Souza W. The peripheral vesicles of trophozoites of the primitive protozoan Giardia lamblia may correspond to early and late endosomes and to lysosomes. J Struct Biol. 1998; 123:225-235. [PubMed: 9878577]

Lindmark DG. Giardia lamblia: localization of hydrolase activities in lysosome-like organelles of trophozoites. Exp Parasitol. 1988; 65:141-147. [PubMed: 3276550]

Nash TE, Aggarwal A, Adam RD, Conrad JT, Merritt JW Jr. Antigenic variation in Giardia lamblia. J Immunol. 1988; 141:636-641. [PubMed: 2454999]

Rivero MR, Miras SL, Quiroga R, Ropolo AS, Touz MC. Giardia lamblia low-density lipoprotein receptor-related protein is involved in selective lipoprotein endocytosis and parasite replication. Mol Microbiol. 2011; 79:1204-1219. [PubMed: 21205007]

Rivero MR, Vranych CV, Bisbal M, Maletto BA, Ropolo AS, Touz MC. Adaptor protein 2 regulates receptor-mediated endocytosis and cyst formation in Giardia lamblia. Biochem J. 2010; 428:3345. [PubMed: 20199400]

Touz, MC. The Unique Endosomal/Lysosomal System of Giardia lamblia. In: Ceresa, B., editor. Molecular Regulation of Endocytosis. Vol. 1. InTech; Croatia: 2012. http://www.intechopen.com/ books/molecular-regulation-of-endocytosis/the-unique-endosomal-lysosomal-system-of-giardialamblia

Touz MC, Lujan HD, Hayes SF, Nash TE. Sorting of encystation-specific cysteine protease to lysosome-like peripheral vacuoles in Giardia lamblia requires a conserved tyrosine-based motif. J Biol Chem. 2003; 278:6420-6426. [PubMed: 12466276]

Touz MC, Nores MJ, Slavin I, Carmona C, Conrad JT, Mowatt MR, Nash TE, Coronel CE, Lujan HD. The activity of a developmentally regulated cysteine proteinase is required for cyst wall formation in the primitive eukaryote Giardia lamblia. J Biol Chem. 2002; 277:8474-8481. [PubMed: 11773053]

Ward W, Alvarado L, Rawlings ND, Engel JC, Franklin C, Mckerrow JH. A primitive enzyme for a primitive cell: the protease required for excystation of Giardia. Cell. 1997; 89:437-444. [PubMed: 9150143] 

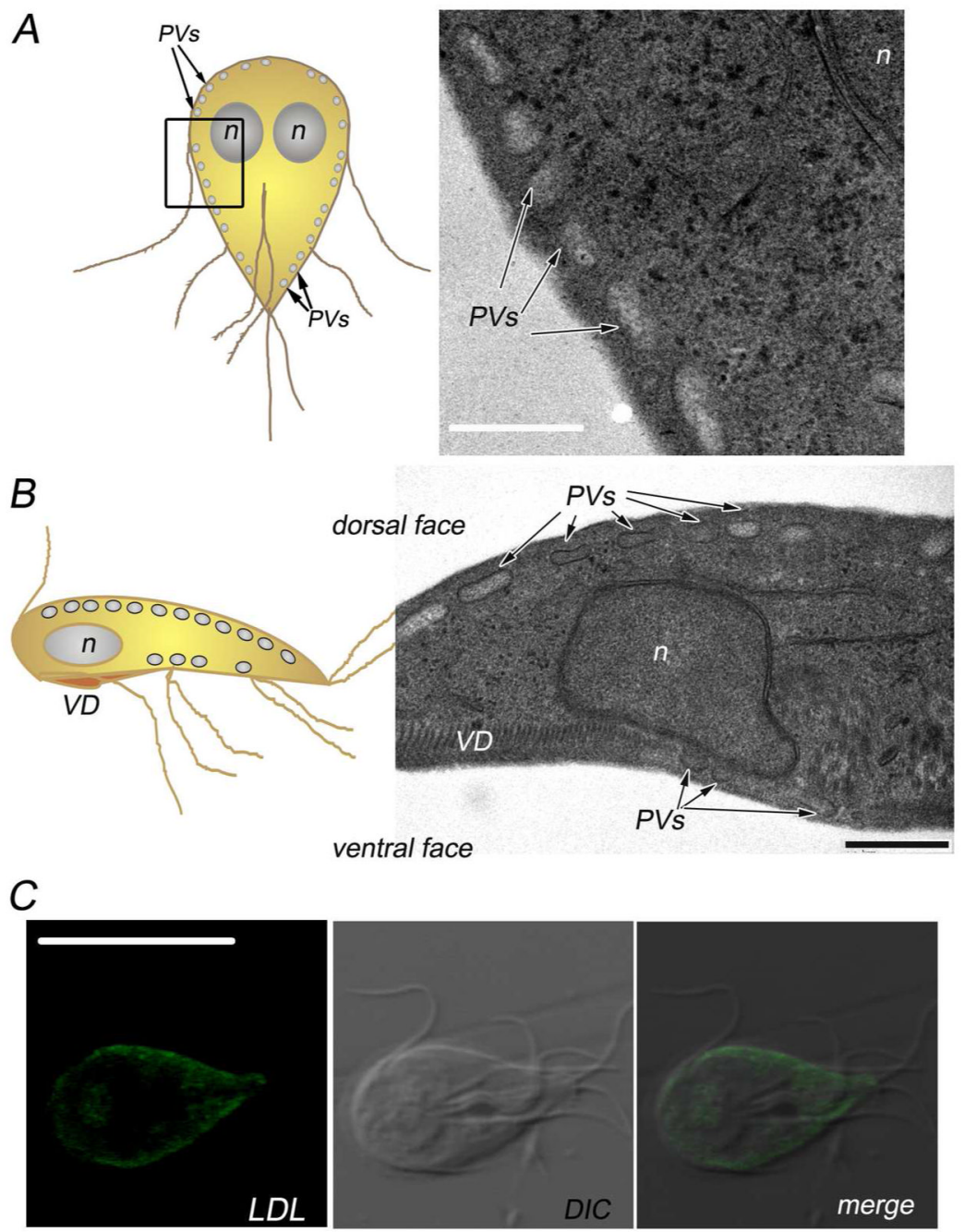

Fig. 1. Endosomal-lysosomal peripheral vacuoles in Giardia lamblia

(A) On the left, cartoon of a Giardia trophozoite. The oval-shape peripheral vacuoles (PVs) are illustrated beneath the plasma membrane. The region analyzed by transmission electron microscopy (TEM) is outlined. On the right, transmission electron microscopy of the region corresponding to the median section, parallel to the dorsoventral axis of the cell, showing the PVs distributed underneath the plasma membrane. n: nucleus. Bar, $500 \mathrm{~nm}$. (B) On the left, cartoon of a trophozoite viewed laterally through the ventral groove. The region showing the array of the PVs analyzed by TEM is bordered. On the right, transmission electron microscopy of a transverse section, showing the PVs distributed below the dorsal and ventral sides of the plasma membrane. n: nucleus. VD: ventral disc (attachment organelle). Bar, $500 \mathrm{~nm}$. (C) BODIPY-LDL fluorescence and confocal microscopy show the distribution of LDL in the PVs underneath the plasma membrane (Rivero et al. 2010). DIC: differential interference contrast microscopy. Bar, $10 \mu \mathrm{m}$. 


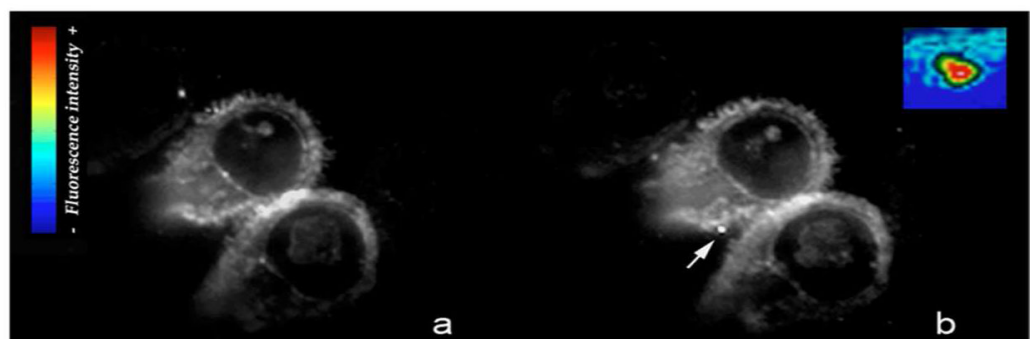

a
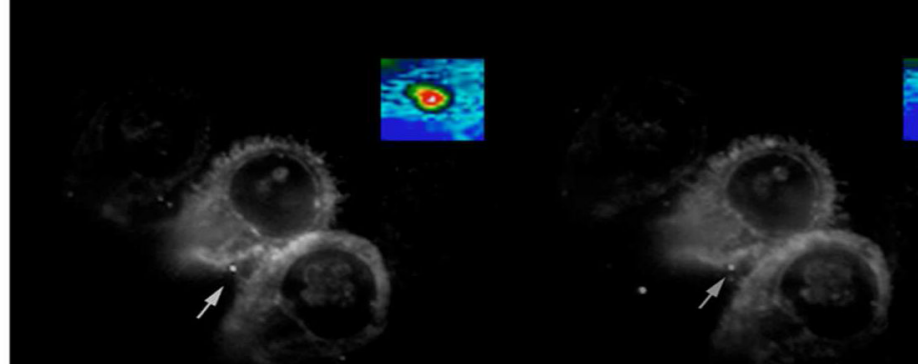

C

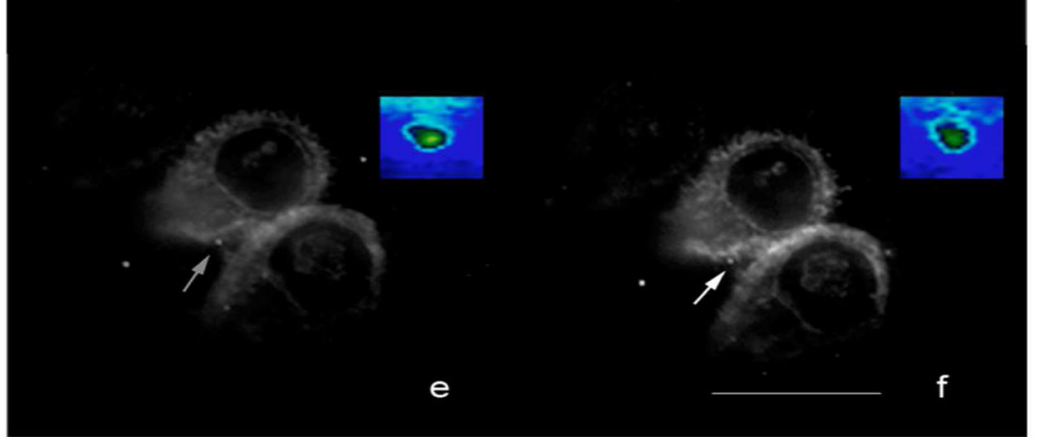

Fig. 2. The capture and internalization of LDL is highly dynamic in living trophozoites TIRF microscopy: Giardia trophozoites were incubated with Bodipy-LDL, and the endocytosis timing was observed. Five min after Bodipy-LDL addition, LDL localized in clusters (a). Cells recorded at an interval of $5 \mathrm{~s} \mathrm{(b} \mathrm{to} \mathrm{f).} \mathrm{Point} \mathrm{of} \mathrm{internalization} \mathrm{of} \mathrm{LDL} \mathrm{is}$ indicated by an arrow. The regions boxed in the TIRF images represent the pseudocolor detail of the LDL internalization (insertions). Pseudocolor bar indicates the surface intensity estimation. Bar $10 \mu \mathrm{m}$. For illustration purposes, one point of internalization is shown. Live images shown represent raw data with simple background subtraction of the averaged blank field intensity. 


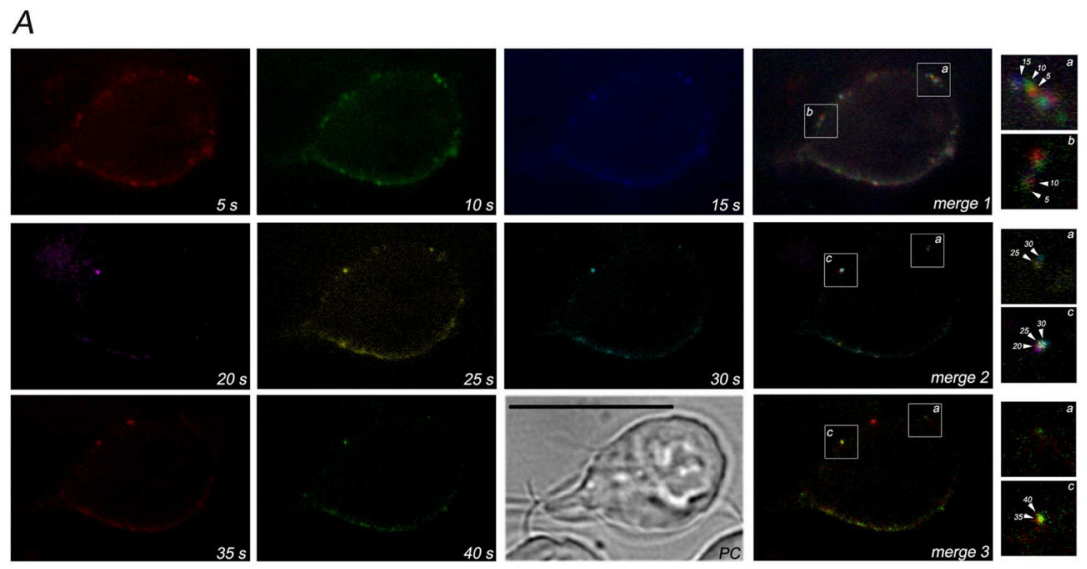

\section{$B$}
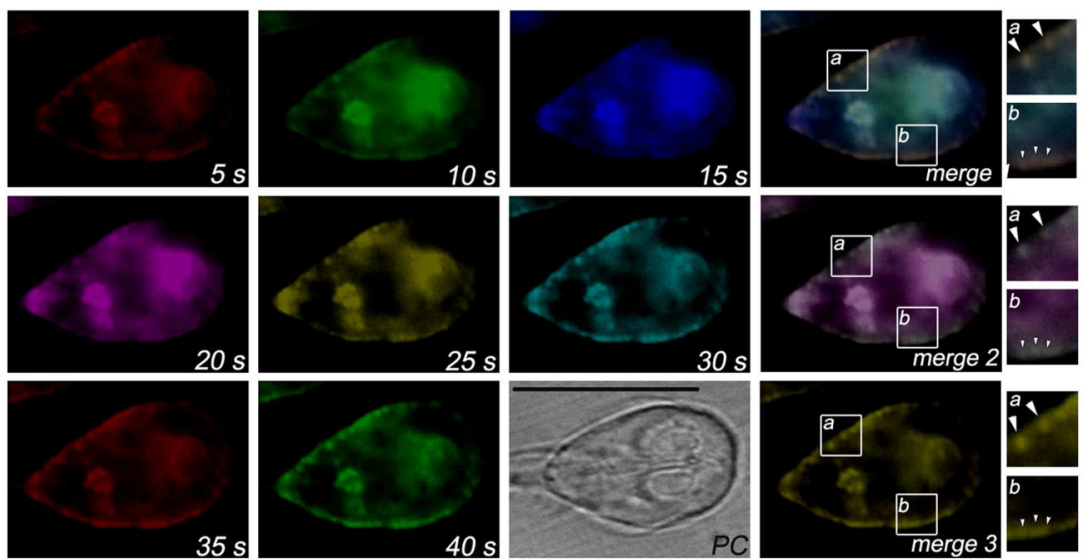

Fig. 3. Time-lapse epifluorescence microscopy shows the internalization and delivery of LDL and dextran

Eight frames from $5 \mathrm{~s}$ to $40 \mathrm{~s}$ were artificially colored using MetaMorph software and combined (merge 1-3) to determine the movement of endocytosed BODIPY-LDL or FITCdextran. (A) The lateral movement of the LDL between the PVs in living cells is observed after following the sequence red, green, blue ( $5 \mathrm{~s}, 10 \mathrm{~s}, 15 \mathrm{~s})$ for merge 1; magenta, yellow, cyan $(20 \mathrm{~s}, 25 \mathrm{~s}, 30 \mathrm{~s})$ for merge 2 ; and red and green ( $35 \mathrm{~s}$ and $40 \mathrm{~s}$ ) for merge 3 . In the insertions $\mathrm{a}, \mathrm{b}$ and $\mathrm{c}$, movement of BODIPY-LDL is observed in detail of seconds (arrowheads). (B) No lateral movement of FITC-dextran between the PVs in living cells is observed after following the sequence red, green, blue ( $5 \mathrm{~s}, 10 \mathrm{~s}, 15 \mathrm{~s})$ for merge 1; magenta, yellow, cyan ( $20 \mathrm{~s}, 25 \mathrm{~s}, 30 \mathrm{~s})$ for merge 2; and red and green (35 s and $40 \mathrm{~s})$ for merge 3 . In the insertions a, b and c, colocalization of FITC-dextran at different time-points is observed in detail (arrowhead). PC: phase contrast. Bars, $10 \mu \mathrm{m}$. 